\title{
Progress of Polymer Materials in Mining and Petroleum Engineering
}

\author{
Hetang Wang $\mathbb{D}^{1},{ }^{1}$ Shengyong $\mathrm{Hu}^{2}$ and Ruiyu Jiang ${ }^{3}{ }^{3}$ \\ ${ }^{1}$ School of Safety Engineering, China University of Mining and Technology, Xuzhou 221116, China \\ ${ }^{2}$ College of Safety and Emergency Management Engineering, Taiyuan University of Technology, Taiyuan 030024, China \\ ${ }^{3}$ School of Chemistry and Chemical Engineering, Yancheng Institute of Technology, Yancheng 224051, China \\ Correspondence should be addressed to Hetang Wang; wanghetang@cumt.edu.cn
}

Received 23 January 2020; Accepted 24 January 2020; Published 18 February 2020

Copyright (c) 2020 Hetang Wang et al. This is an open access article distributed under the Creative Commons Attribution License, which permits unrestricted use, distribution, and reproduction in any medium, provided the original work is properly cited.

Polymer materials are widely used in mining and petroleum engineering, which play an important role in mine and oilfield production. Due to the availability of a variety of polymeric materials, many parameters, such as molecular structure and molecular weight, have substantial effects on their properties. The design and selection of suitable polymers based on the intended specific applications can be challenging. The research into polymeric materials has attracted considerable interest from scientists and engineers in the fields of mining and petroleum. Studies focusing on polymer materials have a wide range of interest and applications in the mining and petroleum industries. This special issue aims to present recent research progress, developments, and applications of polymer materials and technology within mining and petroleum engineering, including theoretical studies, experimental investigations, and research into polymeric applications.

The paper by X. Liu et al. investigated the effect of temperature and accelerator on gel time and compressive strength of the resin anchoring agent. With increasing ambient temperature, the gelation time of the anchorage agent decreased. The peak exothermic value of the anchoring agent grew with the increasing ambient temperature. The compressive strength of resin anchoring agent dropped significantly with the increase in temperature.

K. Gao et al. established a cable combustion experiment platform to study the regularities of the cable fire spread speed and smoke temperature as well as the flame change and molten dripping behaviour. The flame-retardant cable can be ignited and continuously burnt at a certain wind speed, but combustion can be restrained at high wind speed. The combustion speed of the flame-retardant cable is affected by the fire load and ventilation speed. The combustion droplets can change the shape of the flame, which can consequently ignite other combustible materials.

The paper by J. Li et al. proposed that the average formation pressure and its location are changing all the time during alkali-surfactant-polymer (ASP) flooding. In addition, the influence of heterogeneity and viscosity on recovery and pressure is also probed in this paper. A numerical simulation model to match the experiment data considering the physical and chemical alternation in ASP flooding is also developed. Also, the response surface methodology (RSM) is adopted to obtain the formula between pressure functions and influencing factors.

W. Zou et al. investigated the adhesion and adsorption of a hydrophobically modified polyacrylamide (HMPAM) on silica and asphaltene using surface force measurements, thermodynamic analysis, and quartz crystal microbalance with dissipation (QCM-D) measurement. The adhesion force with an HMPAM-modified probe was greater on silica than on the asphaltene surface. Adsorption of HMPAM on the silica surface was greater than that on the asphaltene surface, and the adsorbed layer was more rigid on the silica surface. Hydrophobic interactions between the asphaltene surface and the long hydrocarbon chains of HMPAM may dominate the attraction and adhesion.

Y. Chen et al. proposed a flame retardant with bio-based benzoxazine (Boz-F), red phosphorus, and melamine with a mass ratio of $2: 1: 2$, which then underwent mechanical 
modification by hollow glass bubbles. The pregrouting material with low viscosity and high permeability was compounded, and its interaction with coal was experimentally studied. The new material increases the effective consolidation distance in the coal seam by $40 \%$ on an average compared with traditional polyurethane. Its permeation radius is larger than the calculated radius of the plastic softening zone of a borehole. In addition, the strengths of coal-new material consolidated products with different ratios fully surpass those of coal-polyurethane material consolidated products.

The paper by C. Zhang et al. proposed a new solidified sealing material with better strain-bearing capacity and volumetric expansion capacity. The axial strain and volume increment of the new solidified material is higher than those of the traditional concrete solidified material at the peak stress. Meanwhile, the confining pressure has a certain hysteresis effect on the postpeak stress attenuation. The relative content of seepage pore and fracture in the new solidified material is less than that of coal and concrete samples, and the stress sensitivity of the new solidified materials is weaker than that of coal and concrete materials; hence, new solidified material will have better performance in borehole sealing.

\section{Conflicts of Interest}

The editors declare that they have no conflicts of interest regarding the publication of this special issue.

\section{Acknowledgments}

We would like to express our gratitude to Dr. Taraknath Mandal, Dr. Zhen Li, and all authors who made this special issue possible. We hope this collection of articles will be useful to the scientific community.

Hetang Wang Shengyong $\mathrm{Hu}$ Ruiyu Jiang 